\title{
UTILIZAÇÃO DO PDCA NA GESTÃO DE SEGURANÇA E SAÚDE NO
}

TRABALHO

\author{
USE OF PDCA IN OCCUPATIONAL SAFETY AND HEALTH MANAGEMENT
}

\author{
Ederson Adriano Balduino - edersonbalduino26@bol.com.br \\ Luis Roberto de Medeiros - luis.medeiros@trir.com.br \\ Faculdade de Tecnologia de Taquaritinga (FATEC) - SP - Brasil
}

DOI: 10.31510/infa.v15i1.312

\begin{abstract}
RESUMO
O presente artigo foi elaborado com a finalidade de avaliar a hipótese de utilizar o método PDCA na Gestão de Segurança e Saúde no Trabalho, visando diminuir as vidas perdidas por acidente de trabalho, danos ambientais e patrimonial. A metodologia utilizada foi uma pesquisa bibliográfica, detalhando cada fase do ciclo PDCA voltado à area de Segurança e Saude no Trabalho (SST), descrevendo como fazer o planejamento, como executar o que foi planejado, citando ferramentas necessárias para obter resultados positivos, normas relacionadas à Gestão de (SST), enfatizando o uso da especificação OHSAS 18001:2007, também cita ferramentas que podem ser utilizadas na etapa de verificação, Como a organização deve proceder na etapa Ação do ciclo . E para confrontar com a pesquisa e obtenção de melhor visibilidade do tema no resultado e discussão uma pesquisa aplicada através de uma entrevista com um profissional da área de SST. Relacionando Pesquisa bibliográfica com a entrevista com o profissional da área de Segurança e Saúde no trabalho é possível concluir que o uso do PDCA na área de SST apresenta resultados eficazes, tanto na implementação como na condução da SST.
\end{abstract}

Palavras-chave: PDCA. Segurança e Saúde no Trabalho. OHSAS 18001:2007.

\begin{abstract}
The present article was elaborated with the purpose of evaluating the hypothesis of using the PDCA method in Occupational Safety and Health Management, aiming to reduce the lives lost due to work accidents, environmental damage and property damage. The methodology used was a bibliographical research, detailing each phase of the PDCA cycle focused on the area of Occupational Safety and Health (OSH), describing how to do the planning, how to execute what was planned, citing tools needed to obtain positive results, related standards (OSH) Management, emphasizing the use of the OHSAS 18001: 2007 specification, also cites tools that can be used in the verification step, how the organization should proceed in the Cycle Action step. And to confront with the research and obtain better visibility of the topic in the result and discussion a research applied through an interview with a professional in the area of SST. Relating bibliographical research to the interview with the Occupational Safety and Health professional, it is possible to conclude that the use of PDCA in the area of OSH presents effective results both in the implementation and in the conduction of OSH.
\end{abstract}




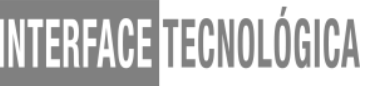

Keywords: PDCA. Safety and Health at Work. OHSAS 18001:2007

\section{INTRODUÇÃO}

Freitas (2016), em seu livro Manual de Segurança e Saúde do Trabalho, cita que no mundo morrem dois milhões de pessoas em decorrência de acidentes de trabalho, acontece também 270 milhões de acidentes de trabalho, e 160 milhões doenças profissionais, diariamente cinco mil pessoas são mortas, esses dados são da OIT. Relacionado a isso esse artigo tem por finalidade, através de uma pesquisa bibliográfica, a utilização do método PDCA na Gestão de Segurança e Saúde no Trabalho, e para melhor visibilidade do tema uma pesquisa aplicada através de uma entrevista com um profissional da área de SST.

O artigo primeiramente traz uma fundamentação teórica do ciclo PDCA, abordando sua criação, definição das quatro etapas, planejamento, execução, checagem, ação e formas de aplicação. Também um histórico de SST no Brasil e no mundo, custo da não-segurança e da segurança. Posteriormente segue o Procedimento metodológicos, nesse trabalho a metodologia utilizada foi detalhar cada fase do ciclo PDCA voltado à area de Segurança e Saude no Trabalho (SST), descrevendo como fazer o planejamento da mesma, o que seguir, quais normas, ferramentas, aqui no Brasil ou no exterior. Como executar dentro do planejado. Como checar, medir, se o planejado e o que foi executado está dentro dos padrões para que possa reiniciar um novo ciclo, replanejar se for necessário. Toda a metodologia foi realizada através de revisão bibliográfica de cada assunto de SST abordado dentro de cada etapa do ciclo PDCA. Que será descrito na seguinte sequência: Etapa P-Planejamento (Plan); Etapa DExecução (Do); Etapa C-Verificação (Check); Etapa A-Ação (Action). Enfatizando o uso da especificação OHSAS 18001:2007. Nos resultados e discussão para avaliar a hipótese de usar esse método, foi realizado uma entrevista com um profissional da área de SST com quarto questões, a primeira um histórico do entrevistado, a segunda sobre sua visão em SST, a terceira seu conhecimento no método PDCA e seu uso em SST, e por fim a quarta foi pedido ao entrevistado que conclua sua visão do tema abordado nesse artigo. Finalizando com uma conclusão dos autores. 


\section{FUNDAMENTAÇÃO TEÓRICA}

\subsection{PDCA}

Andrade (2003), o método PDCA foi criado na década de trinta, nos EUA, por Walter A. Shewhart, estatístico americano, nos laboratórios Bell Laboratóries. Funcionando como um ciclo de controle estatístico do processo. Shewhart publica o livro Economic Control of Quality of Manufactured Product em 1931. Mas, foi popularizado na década de cinquenta pelo especialista em qualidade W. Edwards Deming, passando a ser conhecido mundialmente após sua aplicação nos conceitos de qualidade no Japão. Deming refinou o trabalho de Shewhart, deu o nome de Shewhart PDCA Cycle. Ele alega que não existe metodologia PDCA sem ser definido uma meta a ser atingida. É definido como melhoria contínua.

Deming (1990, apud ALENCAR, 2008), descreve o PDCA em quatro etapas, que são: Plan (Planejamento): consiste no estabelecimento da meta ou objetivo a ser alcançado, e do método (plano) para se atingir este objetivo. Do (Execução): é o trabalho de explicação da meta e do plano, de forma que todos os envolvidos entendam e concordem com o que se está propondo ou foi decidido. Check (Verificação): durante e após a execução, deve-se comparar os dados obtidos com a meta planejada, para se saber se está indo em direção certa ou se a meta foi atingida. Action (Ação): transformar o plano que deu certo na nova maneira de fazer as coisas.

\subsection{Segurança e Saúde no Trabalho, (SST)}

Freitas (2016), em seu livro Manual de Segurança e Saúde do Trabalho, cita que no mundo morrem dois milhões de pessoas em decorrência de acidentes de trabalho, acontece também 270 milhões de acidentes de trabalho, e 160 milhões doenças profissionais, diariamente cinco mil pessoas são mortas, esses dados são da OIT. Ele cita que, há quatro milhões de anos, época dos homens das cavernas, já procedia com segurança, caso contrário a espécie teria sido dizimada, o homem veio se modelando e acomodando-se, ao longo dos séculos.

Pereira (2001), em seu trabalho também cita a Revolução Industrial afetou de forma negativa o bem-estar físico e psicológico do trabalhador, devido ao ambiente inadequado, jornadas de trabalho longas, a falta de habito nas novas máquinas, aumentou o número de acidentes e a primeira lei de proteção do trabalhador foi em 1802 na Inglaterra. No Brasil 
iniciou em 1919. Em 1943 o Código de Legislação Trabalhista-CLT, com função de regulamentar normas trabalhistas, determinando os direitos e deveres do empregador e empregado, tanto a segurança do trabalho, como, salario, jornada, previdência social, aposentadoria.

Oliveira (2003), em seu não obteve resultados positivos, a Segurança e Saúde no Trabalho, não é tratado da forma como deveria tanto pelas empresas como pelos empregados, devido alguns fatores como o baixo envolvimento da alta gestão a esse tema, passando a responsabilidade para os gestores dessa área. Programas nesse princípio de baixo desempenho, privilegiando as situações de risco, falta de cobertura total de fiscalização pelo Ministério do Trabalho. O autor cita sobre o Ato inseguro, não sendo de total responsabilidade do próprio trabalhador, com maior probabilidade ser da organização, por suas falhas de controle que ela exerce sobre o trabalho decorrente do seu sistema de organização, em especial a formalidade do trabalho. Falha nos treinamentos.

No seu trabalho Benite (2004), cita dados do MTE (2003), relacionado com acidentes do trabalho em 2000 no Brasil, foram: 292.793 por Incapacidade Temporária, 11.746 por Incapacidade Permanente, 51.028 por Simples assistência Médica e 2.557 Óbitos. Totalizando 358.124 acidentes no ano.

Segundo dados coletados no Anuário Estatístico da Previdência Social (AEPS, 2015), fonte: DATAPREV, CAT, SUB, no Brasil no ano de 2013 totalizou 725.664 acidente de trabalho, em 2014 - 712.302 e em 2015 foi o total de 612.632 acidentes do trabalho.

Benite (2004, p.22), cita em sua dissertação de mestrado sobre os custos relacionados a não-segurança, ou seja quando a SST não é tratada adequadamente, que são:

- Custo do transporte e atendimento médico do acidentado.

- Prejuízos resultantes dos danos materiais a ferramentas, máquinas, materiais e ao produto.

- Pagamento de benefícios e indenizações aos acidentados e suas famílias.

- Pagamento de multas e penalizações.

- Tratamento de pendencias jurídicas, tais como processos criminais por lesões corporais, indenizatórias e previdenciárias.

- Tempo não trabalhado pelo acidentado durante o atendimento e no período em que fica afastado.

- Tempo despendido pelos supervisores, equipes de SST e médica durante o atendimento.

- Baixa moral dos trabalhadores, perda de motivação e consequente queda de produtividade.

- Tempo de paralisação das atividades pelo poder público e consequente prejuízo à produção.

- Tempo para limpeza e recuperação da área e reinício das atividades.

- Tempo necessário para o replanejamento das atividades.

- Tempo dos supervisores para investigar os acidentes, preparar relatórios e prestar esclarecimentos às partes interessadas: clientes, sindicatos, MTE, imprensa etc. 
- Tempo de recrutamento e capacitação de um novo funcionário na função do acidentado, durante o seu afastamento.

- Perda da produtividade do trabalhador acidentado após o seu retorno.

- Aumento dos custos dos seguros pagos pelas organizações (voluntários e obrigatórios).

- Aumento dos custos para a sociedade, resultante da maior necessidade de recursos financeiros (tributações) para que o governo efetue o pagamento de benefícios previdenciários (auxílio doença, pensões por invalidez etc.), bem como para a manter toda a estrutura existente de fiscalização.

- Custo econômicos relativos ao prejuízo da empresa frente à sociedade e clientes.

Também relatou em sua dissertação os custos da segurança:

- Tempo dos trabalhadores utilizado durante as atividades de treinamento.

- Custo dos treinamentos, conscientização e capacitação dos trabalhadores.

- Custos com exames médicos de monitoramento de saúde.

- Manutenção de equipes de SST e respectivos encargos sociais.

- Aquisição de equipamentos de proteção individual.

- Tempo para desenvolvimento de projetos e instalação de proteções coletivas.

- Placas de identificação e orientativas de SST.

- Manutenção da infra-estrutura nos canteiros (áreas de vivência, refeitórios, alojamento, sanitários).

- Custos com realização de medições de condições ambientais (ruído, iluminação, vapores etc.). (BENITE, 2004, p. 24).

Benite (2004) cita que é comum ter a visão de que os custos da segurança afetam o lucro da empresa mais que os custos da não segurança, mas esse conceito é incorreto, a aplicação da segurança torna a atividade mais eficiente e econômica.

\section{PROCEDIMENTOS METODOLÓGICOS}

Toda a metodologia foi realizada através de pesquisa bibliográfica de cada assunto de SST abordado dentro de cada etapa do ciclo PDCA. Que será descrito na seguinte sequência: Etapa P-Planejamento (Plan); Etapa D- Execução (Do); Etapa C-Verificação (Check); Etapa A-Ação (Action).

\subsection{Etapa P-Planejamento (Plan)}

\subsubsection{OHSAS Occupational Health and Safety Assessment Services 18001:2007}

OHSAS 18001:2007 (2007), Especificação da Série de Avaliação da Saúde e da Segurança do Trabalho, foi desenvolvida pela necessidade de normatização dos Sistemas de Gestão da Saúde e da Segurança do Trabalho podendo avaliar e certificar. Podendo ser aplicada em qualquer organização 


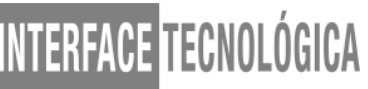

As OHSAS referente à gestão de SST proporciona as organizações os elementos de um Sistema de segurança e saúde do trabalho eficaz, com o intuito de ajudar as organizações à atingir os objetivos de SST. Como outras Normas Internacionais, não pretendem criar barreiras não tarifárias ao comércio nem ampliar ou alterar as obrigações legais de uma organização. O sucesso do Sistema depende do compromisso de todos os níveis e funções da organização, principalmente da Gestão superior. A OHSAS se baseia no ciclo PDCA, na etapa planejar consiste em estabelecer os objetivos e os processos necessários para atingir resultados, de acordo com a política de SST da organização, identificando os perigos, avaliando os riscos e estabelecer medidas de controle. A alta gestão deve definir e autorizar a política de SST.

\subsection{2 (NRs) Normas Regulamentadoras}

As NRs (Normas Regulamentadoras) são obrigatórias pelas empresas privadas e públicas e por órgãos públicos de administração direta ou indireta, com empregados regidos pela CLT, devido ao número de trabalhadores que morreram ou tiveram complicações de saúde em seu ambiente laboral, as NRs foram criadas com o intuito de minimizar, evitar ou extinguir todo e qualquer tipo de acidentes de trabalho. Nesse contexto de atuação essas Normas encaixam na fase de planejamento desse trabalho, pois mesmo sendo de caráter obrigatório no Brasil, é necessário ser levado em questão nessa fase, se não forem aplicadas de forma planejada o resultado pode ser de baixa eficiência. (KOSCHEC; WOLFART; POLACINSKI, 2012).

\subsubsection{Gestão de Riscos}

Em relação ao processo de gerenciamento de riscos Ruppnthal (2013) cita que deve avaliar o contexto da organização, sua atuação, seus objetivos, estratégias, valores, e cultura, com isso estabelece a estrutura que as decisões se apoiam, identifica os perigos, analisa os

riscos quantitativamente ou qualitativamente, estima a ocorrências e impactos, faz o tratamento adequado, modificando o sistema, visando eliminação do risco, atacando os fatores de ocorrência ou consequências, visando reduzir os riscos, se for viável reter o risco com medidas mitigatórias. 


\subsubsection{Plano de Emergência}

Silva e Adissi (2005) citam que mesmo a indústria fazendo esforços para evitar catástrofe é necessário se preparar caso aconteça, através de ações planejadas enfrentará uma emergência de forma mais adequada, evitando improvisos. No acidente ou perigo não é possível estabelecer estratégias considerando todas as consequências, é necessário que a indústria um plano de controle de emergência. A eficácia dos planos pode ser interferida devido alguns fatores, como políticas e programas de SST abstratos, descontextualizados, a elaboração do plano sem a participação dos protagonistas, planos que não atendem a variabilidade organizacional, treinamento, capacitação, dimensionamento de efetivos, organização temporal do trabalho, relacionado as pessoas as competências, variabilidade humana, planos que não evoluem e planos que não são acionados em formas de simulação.

\subsection{Etapa D- Execução (Do)}

A OHSAS 18001:2007 (2007), cita que a organização deve assegurar que todos no seu controle possuam competência adequada para realizar suas atividades, mantendo seus registros associados, as necessidades de formação deve estar associadas com a SST, também deve estabelecer procedimentos com finalidade de sensibilizar as pessoas a atenderem SST.

Cocharero (2007), cita algumas ferramentas que podem ser utilizados na fase de execução para atender SST. Como o Diálogo Diário de Segurança (DDS), consiste em um treinamento diário alternando temas relacionados com a atividade do dia a dia, com a participação de todos, devendo ter uma lista de presença. Inspeção de segurança, tem como objetivo verificar como está a SST no local de trabalho. Cartão do Pare, o trabalhador consegue avaliar se está apto para atividade que irá executar. Investigação de acidentes, é o levantamento de dados sobre o acidente, e possível causa raiz. Análise Preliminar de Risco (APR), tem como objetivo reconhecer e avaliar possíveis riscos na atividade, levantando ações para eliminar ou reduzir a ocorrência deles, o foco dessa ferramenta é eliminar o acidente. Mudança de tecnologia, essa ferramenta consiste em analisar qualquer mudança na organização, sendo no projeto dos equipamentos, processo, insumos, instalações visando a segurança do trabalhador durante e após a mudança.

Segundo Ribeiro e Ferreira (2013) as informações de uma organização devem ser comunicadas adequadamente, na hora e tempo hábil, é fundamental a busca de formas 


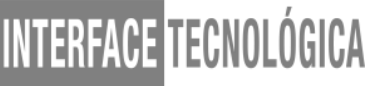

eficazes de comunicação, deste modo, os funcionários bem informados conseguem entender as demandas da organização e aplica-las da melhor maneira. Tendo em vista esse trabalho a segurança e saúde do trabalhador, é de fundamental importância que o sistema de comunicação da organização funcione adequadamente, isso pode ser feito através de reuniões, direcionando a comunicação adequada, interligando a alta gestão com a operação e vice e versa.

No livro facilitadores da Qualidade/ Requisitos e Habilidades do autor Hanlon tradução de Cavalcanti (1995), fala como elaborar reuniões, citando alguns pontos para planejamento de reuniões como: analisar progressos da última reunião, tomar medidas determinadas na última reunião, já planejar a próxima, definir objetivos, definir e seguir a pauta acordando ações, fazer um resumo, emitir atas. Todos os membros devem ser selecionados com base na sua capacidade de contribuírem para a solução do problema.

\subsection{Etapa C - Check (Verificação):}

Segundo a OHSAS 18001:2007 (2007) a organização deve estabelecer e manter procedimentos para monitorar e medir, periodicamente o desempenho em SST, sendo as medidas qualitativas e quantitativas apropriadas, monitorar a extensão em que os objetivos de SST são atingidos, medições proativas do desempenho do programa de gestão em SST, medidas de controle e critérios operacionais, medições reativas do desempenho aos danos acontecidos, como acidentes, quase acidente, registros dos dados de ações preventivas e corretivas. Devendo ser feito auditorias em intervalos planejados.

Uma forma de medir é através de indicadores, segundo Neves (2012) os indicadores de desempenho evoluíram com objetivo de permitir que os gestores tomem decisões baseadas em fatos, pelo motivo de medir diversa variáveis, cita também que cada organização deve implementar um sistema de monitorização, permitindo monitorar o presente e promover medidas de melhorias para o futuro, os indicadores na melhoria contínua apresentam-se como um meio de excelência ao dispor da gestão para monitorar essa melhoria.

Uma ferramenta para medir e gerar ações para prevenir acidentes é a Pirâmide de Frank Bird, com ela é possível levantar as causas antes que aconteça o acidente. Henrique (2013) cita a interpretação dessa pirâmide, para cada acidente sério/Fatal, existem 10 menores (1:10). 30 acidentes com danos materiais (1:10:30), e 600 incidentes (1:10:30:600), ou seja para cada 600 incidentes ou negligência relacionado com algum risco percebido temos um 


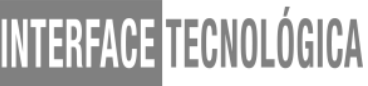

acidente sério ou fatal. Kronemberger (2010) cita em seu trabalho que na base da pirâmide de Bird foi acrescentado comportamentos inseguros.

\subsection{Etapa A- Action (Ação)}

Segundo a OHSAS 18001:2007 (2007) a gestão de topo deve rever o sistema de gestão de SST em intervalos planejados assegurando sua adequação, suficiência e eficácia. Devendo incluir a avaliação de oportunidades de melhorias, necessidade de alteração ao sistema gestão, na política e objetivos de SST, devendo ser mantidos registros de tudo.

É manter o ciclo funcionando, sempre buscando melhorias ao sistema de SST.

\section{RESULTADOS E DISCUSSÃO}

Para avaliar a hipótese de usar esse método, foi realizado uma entrevista com um profissional da área de SST com quatro questões, descrito a seguir:

1- Histórico do Entrevistado:

Antonio Carlos de Sousa Junior é graduado em Engenharia Ambiental e pós-graduado em Engenharia de Segurança do Trabalho. Iniciou a carreira na área de Saúde, Segurança e Meio Ambiente em 2003 em uma empresa multinacional americana líder mundial no segmento e em seguida trabalhou em indústrias de alimentos, indústrias químicas e agronegócios. Atualmente atua na função de Coordenador de Saúde, Segurança e Meio Ambiente.

2- Descreva de acordo com sua visão profissional sobre Segurança e Saúde no Trabalho, (SST), sobre a evolução e limitação dessa área.

Antonio: Considero a área de Saúde e Segurança no Trabalho uma área estratégica, pois o principal foco desta área é assessorar tecnicamente as organizações para que haja a preservação da saúde e integridade física dos seus ativos mais valiosos, seus colaboradores.

3- Em relação ao método PDCA, com sua experiência na área, qual sua opinião do uso desse método na gestão de SST? Poderia dar alguns exemplos?

Antonio: O PDCA é uma metodologia muito eficiente, amplamente utilizada por grande parte das organizações como base de sistemas de gestão. Especificamente quando se trata da gestão de SST, uma norma global de referência para implementação de sistemas de gestão de Saúde e Segurança do Trabalho, que é totalmente baseada na metodologia do PDCA é a atual OHSAS 18001 (Occupational Health and Safety Assessment Series). Em minha 


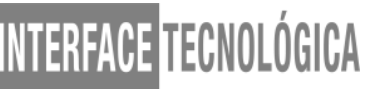

carreira tive a oportunidade de trabalhar em organizações que adotam a OHSAS 18001, como sistema de gestão de SST e também pude vivenciar experiências em implementar sistemas de gestão de SST desenvolvidos pelas próprias organizações, e que podemos notar que o princípio de todos está baseado na metodologia PDCA. Abaixo estou citando alguns exemplos de elementos chaves de sistema de gestão de SST baseado na metodologia do PDCA.

Plan: Definição de uma política de SST; Identificação e avaliação dos riscos de SST; Identificação dos requisitos legais aplicáveis; Definição de objetivos e planos de melhoria. Do: Definição da autoridade e responsabilidades, principalmente da liderança; Gestão de documentos e controle de registros; Preparação e resposta a emergências. Check: Avaliação de requisitos legais e outros requisitos aplicáveis; Avaliação da performance de SST; Investigação de incidentes; Auditoria interna. Act: Revisão do sistema de gestão pela alta administração.

4- Concluindo, qual é sua opinião em relação ao tema abordado nesse artigo?

Antonio: O tema é de suma importância, pois permite obter uma visão mais aprofundada de como a metodologia PDCA pode ser utilizada como base para construção de um sistema de gestão de SST, visando de forma simples e objetiva estruturar a implementação de técnicas de SST e avaliar a eficácia das mesmas em prol da prevenção de incidentes e doenças.

\section{CONCLUSÃO}

Pelo estudo apresentado nesse artigo relacionando com a entrevista com o profissional da área de Segurança e Saúde no trabalho é possível concluir que o uso do PDCA na área de SST apresenta resultados eficazes, tanto na implementação como na condução da SST na organização, com o uso do ciclo é possível gerenciar essa área de forma mais eficaz, agindo mais preventivamente do que corretivamente, planejando suas atividades, executando de forma planejada, checando o planejamento e execução e agindo com melhoria contínua com custo menor que a não segurança, e poupando vidas conforme citado pelo entrevistado os ativos mais valiosos de uma organização são seus colaboradores. Mas para obter resultado positivo deve partir do alto nível da organização atingindo todos os níveis. 


\section{REFERÊNCIAS}

AEPS-ANUÁRIO ESTATISTICO DA PREVIDÊNCIA SOCIAL. V. 24. Brasília, 2015. Disponível em: <http://www.previdencia.gov.br/wp-content/uploads/2015/08/AEPS-2015FINAL.pdf $>$. Acesso em: 09 abr. 2017.

ALENCAR, J, F. Utilização do Ciclo PDCA para Análise de Não Conformidades em um Processo Logístico. Monografia-Universidade Federal de Juiz de Fora. Juiz de Fora-MG, 2008. Disponível em: 〈http://www.ufjf.br/ep/files/2014/07/2008_3_Joana.pdf〉. Acesso em: 01 mar. 2017.

ANDRADE, F, F. O Método de Melhorias PDCA. Dissertação Apresentada à Escola Politécnica da Universidade de São Paulo para a Obtenção do Título de Mestre em Engenharia. São Paulo, 2003. Disponível em: <file:///C:/Users/Adm/Downloads/dissertacao_FABIOFA\%20(1).pdf〉. Acesso em: 18 jan. 2017.

BENITE, A, G. Sistema de Gestão da segurança e Saúde no Trabalho para Empresas Construtoras. Dissertação Apresentada à Escola Politécnica da Universidade de São Paulo para a Obtenção do Título de Mestre em Engenharia. São Paulo, 2004. Disponível em:<file:///C:/Users/Adm/Downloads/AndersonBenite\%20(1).pdf〉. Acesso em: 18 jan. 2017.

COCHARERO, R. Ferramentas para Gestão de Segurança e Saúde do Trabalho no Canteiro de Obras. Monografia Apresentada à Escola Politécnica da Universidade de São Paulo para a Obtenção do Título de MBA em Tecnologia e Gestão na Produção de Edifícios. São Paulo, $2007 . \quad$ Disponível em: <http://www.pcc.usp.br/files/text/personal_files/francisco_cardoso/Monografia\%20\%20Renato\%20Cocharero\%20_TGP\%20-\%202007_\%20Final.pdf>. Acesso em: 19 jan. 2017.

FREITAS, L, C. Manual de Segurança e Saúde do Trabalho. Edições Sílabo, $3^{\circ}$ Edição, Cap. 1, Lisboa, 2016. Disponível em: 〈http://www.silabo.pt/Conteudos/8667_PDF.pdf>. Acesso em: 18. jan. 2017.

HANLON, T. Facilitadores da Qualidade: requisitos e habilidades. Traduzido por CAValCANTI, M, F, H. Pioneira-São Paulo. Grifo Enterprises-Rio de Janeiro, 1995. p. 5 à 60.

HENRIQUE, L, Pirâmide de Frank Bird. Insight em Saúde e segurança do Trabalho, 2013. Disponível em: <http://insightemsst.blogspot.com.br/2016/05/piramide-de-frank-bird.html>. Acesso em: 15 ago. 2017.

KOSCHEC, D; WOLFART, T; POLACINSKI, È. Normas Regulamentadoras no Contexto da Segurança do Trabalho: uma Abordagem Conceitual. $2^{\mathbf{0}}$ Semana Internacional das Engenharias da FAHOR. Faculdade Horizontina. Horizontina, RS, Brasil. 22 a 26 de 
Outubro

de

2012.

Disponível

em:

<http://www.fahor.com.br/publicacoes/sief/2012_22.\%20NORMAS\%20REGULAMENTAD ORAS\%20NO\%20CONTEXTO\%20DA\%20SEGURAN\%C3\%87A\%20DO\%20TRABALH O\%20-\%20UMA\%20ABORDAGEM\%20CONCEITUAL.pdf>. Acesso em: 18 jan. 2017.

KRONEMBERGER, P, R, M. O Novo Programa de Comportamento Seguro da Votorantim Metais Zinco de Juiz de Fora. Trabalho de conclusão de curso (Graduação em Engenharia de Produção) -Universidade Federal de Juiz de Fora, Juiz de Fora. Juiz de Fora-MG, 2010. Disponível em: <http://www.ufjf.br/ep/files/2014/07/2010_3_Pedro-Roberto.pdf>. Acesso em: 15 ago. 2017.

NEVES, A. O Uso de Indicadores Chave de Desempenho para Avaliar a Eficiência dos Sistemas de Gestão. ISEC-Instituto Superior de Educação e Ciências, 2012. Disponível em:

<http://comum.rcaap.pt/bitstream/10400.26/8978/1/Disserta\%C3\%A7\%C3\%A3o\%20Mestrad o_Andreia\%20Neves\%20KPI.pdf >. Acesso em: 26 jan. 2017.

OHSAS 18001:2007. Sistema de Gestão da Segurança e da Saúde do Trabalho-Requisitos. Disponível em: $<$ http://comum.rcaap.pt/bitstream/10400.26/7319/2/Anexo\%20I\%20OHSAS180012007_pt.pd f>. Acesso em: 05 mar. 2017.

OLIVEIRA, J, C. Segurança e Saúde no Trabalho uma Questão Mal Compreendida. São Paulo em Perspectiva. 2003. Disponível em: <http://www.scielo.br/pdf/spp/v17n2/a02v17n2.pdf>. Acesso em: 18 jan. 2017.

PEREIRA, V, T. A Relevância da Prevenção do Acidente de Trabalho para o Crescimento Organizacional. Universidade da Amazônia-UNAMA, Centro de Ciências Humanas e Educação-CCHE, Curso de Serviço Social. Belém-Pará. 2001. Disponível em: <http://www.segurancaetrabalho.com.br/download/prev-vandilce.pdf $>$. Acesso em: 18 jan. 2017.

RIBEIRO, A, E, L; FERREIRA, V, C, P. O Sistema de Comunicação Interna de uma Indústria, na Percepção de seus Funcionários. Gestão e Tecnologia para a Competitividade, 2013. Disponível em: <http://www.aedb.br/seget/arquivos/artigos13/49418571.pdf>. Acesso em: 25 jan. 2017.

RUPPENTHAL, J, E. Gerenciamento de Riscos. Universidade Federal de Santa Maria, Colégio Técnico Indústrial de Santa Maria, Rede e-Tec Brasil. Santa Maria-RS. 2013. Disponível em: <http://estudio01.proj.ufsm.br/cadernos_seguranca/sexta_etapa/gerenciamento_riscos.pdf $>$. Acesso em: 19 jan. 2017.

SILVA, R, L, A; ADISSI, P, J. Plano de Controle de Emergência: um Roteiro para Elaboração. XXV Encontro Nac. de Eng. de Produção. Porto Alegre-RS, 2005. Disponível 


\section{INTERFAGETECENOLOGGCA}

em: $\quad<$ http://www.factor-segur.pt/wp-content/uploads/2014/11/Planos-de-Emergencia-naIndustria.pdf $>$. Acesso em: 23 jan. 2017. 